\title{
Question and Answer Sessions with Dr. George O. Waring, III
}

\section{Tokyo}

\section{Chairman: Dr. Mitsuru Sawa \\ (Nihon Univ.)}

Dr. Sawa: I understand that refractive surgery is a staged surgery. Surgery with phakic IOLs can give us good refractive correction. But, there have been some reports that eyes with phakic IOLs have persistently raised flare values. This means that the phakic IOL affects the flare value. What do you think of these aspects of physiology after phakic IOL surgery?

Dr. Waring: That is an excellent question, because every style of phakic intraocular lens that we use today has reports in the literature of serious complications: cataracts induced with the posterior chamber intraocular lens; chronic flare and cells with the iris-fixated intraocular lens, the Artisan; retraction of the iris with oval pupils; or damage to the endothelium with the angle-fixated intraocular lens. But, the most important thing to realize is that every style of intraocular lens has undergone many changes in design. And, we must not make the mistake of saying that the complications with early models are the same as the complications with the present models. Most of the things that I have just mentioned no longer exist, or exist rarely, with the current models of these phakic intraocular lenses. Specifically, the question of intraocular inflammation was reported in the early designs of the Worst iris-claw lens and of the angle-fixated lenses, but is not in the present designs.

Dr. Shimizu (Kitazato University): My name is Shimi$\mathrm{zu}$ and I work in Tokyo. I have two questions. One ques- tion regards phakic eyewear. I have been confronted with 50 cases of PIOL over the last 5 years. The most difficult aspect is determining what length is desirable for the individual eye. It is very difficult to measure sulcus to sulcus, because it is not related to white to white. So my question is how to accurately measure sulcus to sulcus?

Dr. Waring: Which intraocular lens style did you implant?

Dr. Shimizu: The STAAR.

Dr. Waring: The STAAR lens. This is an excellent question for posterior chamber lenses and for anterior chamber lenses. The white-to-white measurement is inaccurate with a variability of at least $1 \mathrm{~mm}$. So, some lenses will be too big, and some lenses will be too small. The solution to that is to use $50-\mathrm{MHz}$ high-resolution ultrasound to image the anterior segment. And such an instrument is now commercially available. That is the only way that I know to measure the sulcus and the anterior chamber today. This is called the 'Artemis' instrument and it is available from Florida in the United States. This is one reason that I personally like the Artisan lens, because one size fits all. It is $8.5 \mathrm{~mm}$ in its long diameter and it has a 6-mm optic. Then, you do not have to worry about trying to measure the white to white. Did you have a second question?

Dr. Shimizu: Yes, I have a second question. I agree that bioptics is a very wonderful technique. But, I have some trouble. At first, I used cataract surgery. After that, I used the wavefront instrument to measure the eye with eyewear. And I performed wavefront LASIK after the cataract surgery. But this is not correct, because it is very diffi-

\footnotetext{
KARGER

Fax +4161306 1234

E-Mail karger@karger.ch

www.karger.com

(C) 2003 S. Karger AG, Basel

0030-3755/03/2177-0045\$19.50/0

Accessible online at:

www. karger.com/oph
} 
cult to measure the wavefront in the eye with eyewear. The normal eye gives very accurate measurements, but with the eyewear, the pseudophakic eye, it is very difficult to conduct the wavefront analyses. So, how would you avoid these problems?

Dr. Waring: We have a wonderful opportunity tonight, because Dr. O'Brien is still here. So why don't I ask him to come here to help comment on this. One approach to that is to use topographically guided treatment. That is, to use the anterior surface of the cornea as the basis for the treatment and not the entire wavefront in the eye. This will allow us to avoid the problems that are induced by the intraocular lens and still correct most of the aberrations which still come from the cornea. That would be one approach.

Dr. O'Brien: I think the different types of wavefront analyzers are advancing. And, we do have difficulty, especially with the outgoing Hartmann-Shack type wavefront analyzers in the pseudophakic eye. However, with the ingoing or the retinal ray-tracing type, like the Tracey System, we have been able to capture images in pseudophakic eyes with a higher degree of accuracy and precision. So, I do think advances in wavefront sensing using different aberrometers than the Hartmann-Shack type may be a helpful solution in the future.

Dr. Waring: There are three aberrometers that are available now in what we would call 'single pass'. The Tscherning Aberrometer, the Tracey ray-tracing system, and at Emory, we have the InterWave System, which is actually the only subjective instrument because this involves the patient actually responding to each point that is tested, so that gets the occipital cortex involved as well. It may be that using single pass systems, we can reduce the amount of noise that is introduced by the intraocular lens itself. Other questions?

Dr. Sawa: No more questions? It is time to close this session. Thank you very much Dr. Waring and Dr. O'Brien.

\section{Osaka}

Chairman: Dr. Shigeru Kinoshita

(Kyoto Univ. of Medicine)

Dr. Kinoshita: Thank you Dr. Waring for that comprehensive lecture on this theme. Does anybody in the audience have a question? If there are none, let me ask a question. Phakic IOLs are classified into anterior chamber lenses and posterior chamber lenses. Anterior chamber lenses raise concern about the corneal endothelium.
Posterior chamber lenses involve a risk of cataract as part of the long-term prognosis, although the occurrence of this complication has decreased considerably. Dr. Waring, what is your opinion on these points?

Dr. Waring: Yes, I think that your concerns are 100\% correct. There is no intraocular lens that we can implant that is completely safe. But, it is very important to remember that all of the phakic intraocular lenses have gone through three or four different changes in design. That's true for the anterior chamber angle-fixated lenses, for the iris-fixated lens, the 'Artisan', and for the posterior chamber placed lenses. And, we must not confuse the complications that occurred in the early designs with the present complication rate. There was endothelial damage earlier. These induced cataracts earlier. There was chronic iridocyclitis in all of these early models. But, we are lucky that the surgeons and the companies did not stop developing the designs. Today, we find a very low complication rate with the current models of these lenses. They are much safer than they were 2 or 3 years ago.

Dr. Kinoshita: Let me ask another question connected to the first one. If a patient with approximately $-10 \mathrm{D}$ is considered a borderline candidate for simple LASIK based on having a relatively thin cornea, do you perform combined/staged surgery with phakic IOLs followed by LASIK, or do you still prefer conventional LASIK?

Dr. Waring: I am glad you are paying attention. The approach that I take at Emory in the United States is that in any myope that has more than $9 \mathrm{D}$ of myopia, I place a phakic intraocular lens, no matter what the corneal thickness is. And then, I will go back and do a LASIK adjustment if necessary. I personally believe that the standard for phakic intraocular lens placement in both hyperopes and myopes will be coming down to $9 \mathrm{D}, 8,7,6$, because the optics of an intraocular lens are superior to the optics that we create in the cornea today. And, you can see this in Latin America and Europe where these lenses are commonly used. They are being used for lower amounts. And probably the best cut for LASIK using today's technology is around 5 or $6 \mathrm{D}$, and phakic intraocular lenses are preferable for greater amounts.

Dr. Kinoshita: It seems that we have gained a great deal of confidence in the use of phakic IOLs. Now, this is my last question. Which do you prefer, anterior chamber or posterior chamber phakic IOLs? This may be a difficult question, because there are various manufacturers and materials.

Dr. Waring: Remember, there are three: AC, PC, and the iris-claw lens of Worst. My preference today is the irisclaw lens for one main reason: one size fits all. When we
46

Ophthalmologica 2003;217(suppl 1):45-47
Question and Answer Sessions with Dr. George O. Waring, III 
place an anterior chamber angle-fixated lens or a sulcusfixated lens, we need to know the proper diameter. Measuring white to white is not very accurate. So, we will always have a few lenses that are too big or too small, when we place anterior chamber or sulcus posterior chamber intraocular lenses. With the Ophtec Artisan lens, the $8.5-\mathrm{mm}$ diameter can fit in essentially all eyes without worrying about proper sizing. The honest answer to your question is that intraocular lens designs for phakic eyes are changing so much that our responsibility is to keep up with that and not to say 'I like the anterior chamber' or 'I like the posterior chamber', but to be alert to the creativity of ophthalmic surgeons and of manufacturers, because there will be much better designs that will come along with time.

Dr. Kinoshita: Dr. Waring prefers iris-claw lenses mainly because one size fits all, but as he remarked, we must not jump to conclusions. We must be alert to future developments, since IOL designs for phakic eyes are changing. While Japanese ophthalmologists have just started clinical trials for phakic IOLs, Dr. Waring's comments made us realize that these lenses are attracting considerable attention in the United States as a key technology in refractive surgery. It is time to close this session. Thank you Dr. Waring. 